\title{
Characterizing and Quantitating Therapeutic Tethered Multimeric Antibody Degradation Using Affinity Capture Mass Spectrometry
}

Wenjing Li*, Neha Srikumar*, William F. Forrest, Diego Ellerman, Chen Gu, Robert Tchelepi, Greg A. Lazar, Yichin Liu, John C. Tran

*These authors contributed equally; \#Author Correspondence: john.tran@gene.com

Genentech, 1 DNA Way, South San Francisco, CA 94080

The supporting information enclosed include:

(1) Supplementary Figure 1

(2) Supplementary Figure 2

(3) Supplementary Table 1

(4) Supplementary Table 2

Additional Supplementary Files also include a proposed script that incorporates the formatted data for generating the fit model of the calibration curves, and the back-calibrations used in the manuscript. These additional files include:

(I) dat_IgG_3FAb_type_01.csv, containing the data file underlying Figure 3A.

(II) dat_IgG_3FAb_type_02.csv, containing the data file underlying Figure 3B.

(III) calibrate_mass_spec_IgG_FAb3.R, containing the code that read the two CSV files (I, II) resulting in the plots for Figure 3. Executing the file produced the files IV, V, and VI.

(IV) "fraction_2_FAb_with_standard_curves.pdf", containing the figures of all the raw standard data and fitted 5-parameter logistic standard curves.

(V) "five_parameter_model_summaries_IgG_FAb3.txt"', containing the parameter fits output for the two data sets (I and II).

(VI) "back_calibrated_IgG_FAb3.csv", containing the data of the two input files (I, II) with the right-most column consisting of the measured degradations interpolated from the calibration curves for both the in vitro and in vivo stability studies. 
Raw Intensities for Fab-IgG I Intact and Product Species

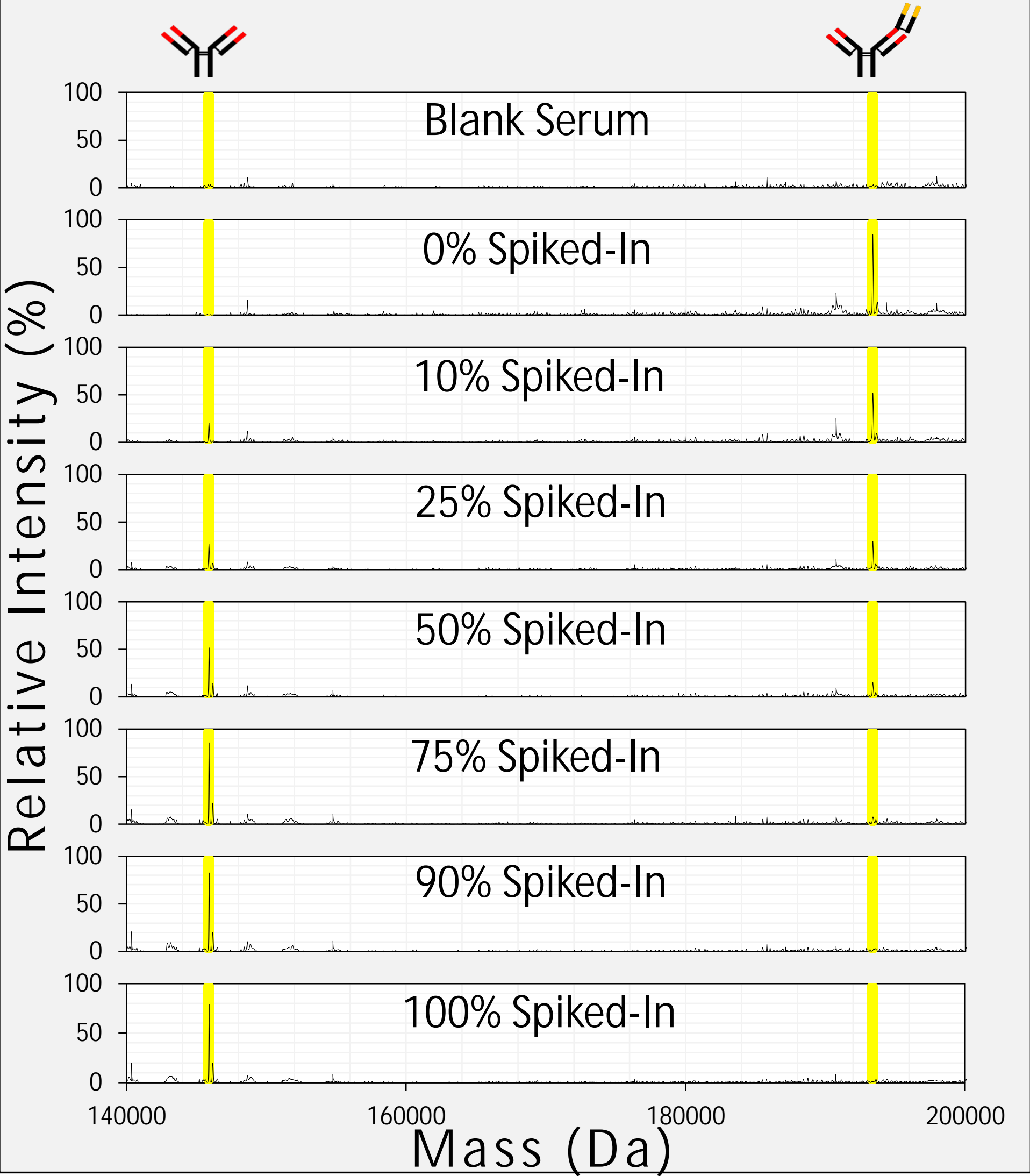

Supplementary Figure 1. Deconvoluted MS data (1 out of 3 replicates) revealing the relative intensities of the spiked-in predicted degraded product (IgG) and stable multivalent Fab-IgG I at varying percentage. The specified percentage is the composition of degraded product relative to the total concentration of both species. 
Raw Intensities for Fab-IgG II Intact and Product Species

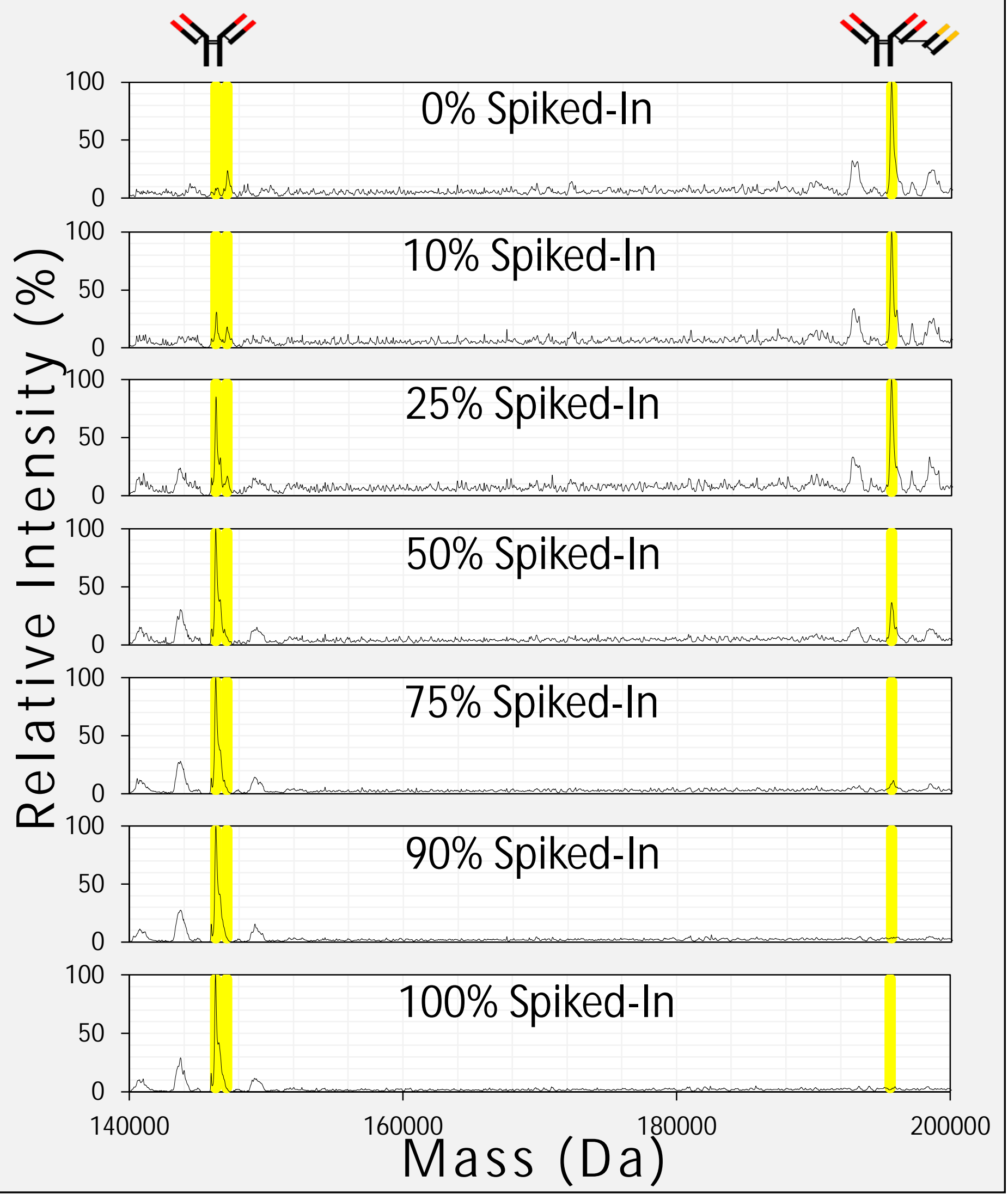

Supplementary Figure 2. Deconvoluted MS data (1 out of 3 replicates) revealing the relative intensities of the spiked-in predicted degraded product (IgG) and stable multivalent Fab-IgG II at varying percentage. The specified percentage is the composition of degraded product relative to the total concentration of both species. 
Supplementary Table 1. Parameter estimates for five-parameter logistic fit (equation 2) for Fab-IgG I and Fab-IgG II calibration curves. Standard error, t-values, and p-values for each parameter fit is reported alongside total residual standard error for the fit.

\begin{tabular}{|c|c|c|c|c|c|}
\hline & Parameter & Estimate & $\begin{array}{c}\text { Standard } \\
\text { Error }\end{array}$ & t-value & p-value \\
\hline \multirow{4}{*}{ Fab-IgG I } & $\mathrm{b}$ & -3.67589 & 1.27016 & -2.894 & 0.01057 \\
\cline { 2 - 6 } & $\mathrm{c}$ & 0.022269 & 0.014099 & 1.5795 & 0.13378 \\
\cline { 2 - 6 } & $\mathrm{d}$ & 1.025598 & 0.038936 & 26.3406 & $1.33 \mathrm{E}-14$ \\
\cline { 2 - 6 } & $\mathrm{e}$ & -0.47512 & 0.084114 & -5.6486 & $3.63 \mathrm{E}-05$ \\
\cline { 2 - 6 } & $\mathrm{f}$ & 0.230829 & 0.096409 & 2.3943 & 0.02925 \\
\hline \multicolumn{5}{|l|}{} \\
\hline \multirow{5}{*}{ Residual Standard error: 0.024 (16 degrees of freedom) } \\
\hline \\
\cline { 2 - 6 }
\end{tabular}


Supplementary Table 2. Precision and Accuracy Results for IgG and Fab-IgG II spiked-in quality control samples.

\begin{tabular}{|c|c|c|c|}
\hline $\begin{array}{c}\text { IgG Spiked-In } \\
(\%)\end{array}$ & $\mathbf{5 \%}$ & $\mathbf{1 5 \%}$ & $\mathbf{2 0 \%}$ \\
\hline $\begin{array}{c}\text { Relative Ion } \\
\text { Response \% } \\
\text { (n =3) }\end{array}$ & 3.2 & 15.8 & 20.0 \\
\hline Average \% & 5.2 & 15.9 & 19.3 \\
\hline \% Bias & -9.1 & 14.2 & 19.4 \\
\hline \% CV & 26.5 & 15.3 & 19.5 \\
\hline
\end{tabular}

